\title{
EFECTO DEL USO COMBINADO DE LA RADIACIÓNUV-CY ATMÓSFERA MODIFICADA SOBRE EL TIEMPO DE VIDA ÚTIL DE UVILLA (PHYSALIS PERUVIANA L.) ORGÁNICA
}

Autoras:

Carlota Moreno Guerrero' María José Andrade Cuvi' Analía Concellón ${ }^{2,3}$

1 Universidad Tecnológica Equinoccial, Centro de Investigación Facultad de Ciencias de la Ingeniería, Laboratorios de Química y Microbiología de Alimentos. Av. Occidental y Mariana de Jesús, CP EC170129 Quito-Ecuador. *cmoreno@ute.edu.ec

2 Centro de Investigación y Desarrollo en Criotecnología de Alimentos (CIDCA). CCT La Plata, CONICET-UNLP. Calle 47 esq. 116. CP 1900. La Plata, Argentina.

3 Comisión de Investigaciones Científicas de la Provincia de Buenos Aires (CIC-PBA)

REVISTA DE INVESTIGACIÓN CIENTÍFICA 



\section{Resumen}

El objetivo del presente trabajo de investigación fue evaluar el efecto del uso combinado de la radiación UV-C y atmósfera modificada sobre el tiempo de vida útil de uvilla orgánica sin capuchón. Se empleó uvilla orgánica producida en una parcela de la Unión de Organizaciones Campesinas e Indígenas de Cotacachi - UNORCAC, Provincia de Imbabura. Los frutos se seleccionaron y lavaron. Para la determinación de la dosis de radiación UV-C, fruta tratada ( 8 y $12 \mathrm{~kJ} / \mathrm{m} 2$ ) y control se almacenó a $5^{\circ} \mathrm{C}$. Cada 7 días se evaluó el avance del daño mediante el valor de índice de daño, pérdida de peso y firmeza. Una vez seleccionada la dosis, se combinó la radiación UV-C con empaque en atmósfera modificada AM (5\% CO2-5\% O2 y 5\%CO2-10\% O2) y se determinó cada 7 días: pérdida de peso, índice de daño y color superficial. Se seleccionó la dosis de 12 $\mathrm{kJ} / \mathrm{m} 2$ porque produjo menor pérdida de peso, índice de daño y ablandamiento, permitiendo mantener la calidad comercial de los frutos por más tiempo (durante 28 días) que los frutos control y tratados con $8 \mathrm{~kJ} / \mathrm{m} 2$. El uso combinado de la radiación UV-C (12 kJ/m2) y el empaque en atmósfera modificada (5\% $\mathrm{CO}_{2}-5 \% \mathrm{O} 2$ y 5\% $\left.\mathrm{CO}_{2}-10 \% \mathrm{O} 2\right)$ redujo la pérdida de peso e índice de daño y produjo ligeros cambios en los parámetros L* y Hue de la uvilla. La combinación UV-C (12 kJ/m2) y 5\%CO2-5\%O2 retrasó la aparición de síntomas de daño permitiendo incrementar la vida útil de la uvilla hasta 35 días.

Palabras clave: uvilla, radiación UV-C, poscosecha, atmósfera modificada.

\section{Abstract}

The objective of this research was to evaluate the effect of the combined use of UV-C and modified atmosphere on the shelf life of organic uvilla uncapped. Organic uvilla produced was used on a plot of the Union of Peasant and Indigenous Organizations of Cotacachi - UNORCAC, Imbabura province. The fruits were selected and washed. For the determination of the dose of UV-C, treated fruit $(8 \mathrm{and} 12 \mathrm{~kJ} / \mathrm{m} 2)$ and control was stored at $5{ }^{\circ} \mathrm{C}$. Every 7 days the progression of damage assessed by the index value of damage, loss of weight and strength. Once selected dose combined UV-C radiation with modified atmosphere packaging AM (5\% $\mathrm{CO}_{2}-5 \% \mathrm{O}_{2}$ and $\left.5 \% \mathrm{CO}_{2}-10 \% \mathrm{O}_{2}\right)$ and determined every 7 days: weight loss, damage index and surface color. We selected a dose of $12 \mathrm{~kJ} / \mathrm{m} 2$ because it produced less weight loss, and damage softening rate, thereby maintaining the commercial quality of the fruit for longer (for 28 days) than control fruit and treated with $8 \mathrm{~kJ} / \mathrm{m} 2$. The combined use of UV-C (12 kJ/m2) and modified atmosphere packaging ( $5 \% \mathrm{CO}_{2}-5 \% \mathrm{O} 2$ and $\left.5 \% \mathrm{CO} 2-10 \% \mathrm{O} 2\right)$ reduced weight loss and rate of damage and produced light changes in the parameters $\mathrm{L}$ * and Hue of uvilla. The combined UV-C $(12 \mathrm{~kJ} / \mathrm{m} 2)$ and $5 \% \mathrm{CO}_{2}-5 \% \mathrm{O} 2$ delayed the onset of symptoms of damage allowing increased uvilla life up to 35 days.

Keywords: uvilla, UV-C, postharvest, modified atmosphere. 


\section{Introducción}

La uvilla (Physalis peruviana), es una fruta climatérica, no tradicional, originaria de los Andes sudamericanos (Perú, Ecuador, Colombia y Bolivia) (Sánchez, 2002). Se caracteriza por su fruto anaranjado (baya carnosa), cubierto por un cáliz que lo protege de patógenos, insectos y condiciones ambientales adversas (CORPEI, 2009). Las condiciones óptimas de cultivo son: una altura entre 1000 y 3000 m.s.n.m. con temperaturas promedio de 14 a $18^{\circ} \mathrm{C}$. La producción nacional de uvilla, en el Ecuador, registra un crecimiento de expansión constante, encontrándose la mayor concentración de plantaciones comerciales en la Provincia de Cotopaxi (para el mercado nacional e internacional), existen también importantes áreas cultivas en Tungurahua, Imbabura y Pichincha (Brito, 2002). La demanda nacional está creciendo, porque ahora se tiene la fruta fresca en los supermercados (PROFIAGRO, 2007).

Los problemas más característicos que aceleran el deterioro de la uvilla incluyen: rajaduras, hongos en el cáliz, hongos en el fruto, ablandamiento, pudrición y cambios sensoriales (Herrera, 2003), de ahí la necesidad proponer alternativas tecnológicas que permitan reducir las pérdidas poscosecha de uvilla.

Las condiciones óptimas de almacenamiento durante el transporte son $4-8{ }^{\circ} \mathrm{C}$ con una humedad relativa del $80-90 \%$. Temperaturas menores y largo tiempo de exposición pueden generar daños por frío, cuyos síntomas son presencia de pudriciones, pérdida de la calidad visual, decoloraciones, entre otros (García, Peña, \& García, 2008).

En algunos estudios sobre tratamientos de luz UV-C se ha demostrado que ésta se puede utilizar para controlar varias enfermedades de frutas y hortalizas originadas por mohos (Stevens et al., 1997) y ha resultado un sistema efectivo para prolongar la vida útil de estos productos por ser letal para la mayoría de microrganismos (Baka, Mecier, Corcuff, Castaigne, \& Arual, 1999)
Por otro lado, el almacenamiento en atmósfera modificada (AM) es una técnica física que no deja residuos químicos en los productos y tiene como objetivo principal cambiar el microambiente del producto almacenado y se fundamenta en la variación del contenido de oxígeno, anhídrido carbónico o nitrógeno en las cámaras de almacenamiento (López \& Páez, 2002) y ha sido ampliamente estudiado y aplicado, con buenos resultados en la extensión de la vida útil de productos frutihortícolas, así como en control del crecimiento de microorganismos durante el almacenamiento refrigerado.

Se ha comprobado en varios productos fruti-hortícolas, que la aplicación de radiación UV-C o de atmósferas modificadas ha inhibido el desarrollo de podredumbres y ha prolongado la vida de anaquel. Pero hasta ahora se desconoce el efecto de la radiación UV-C combinada con atmósfera modificada, sobre calidad poscosecha de frutos y hortalizas.

El objetivo del presente trabajo fue estudiar el efecto de la aplicación de radiación UV-C en combinación con atmósfera modificada en el deterioro de uvilla (Physalis peruviana) orgánica.

\section{Materiales y Métodos}

\section{Material Vegetal}

Se empleó uvilla orgánica producida en el Cantón Cotacachi, Provincia de Imbabura (Ecuador), en una parcela perteneciente a la Unión de Organizaciones Campesinas e Indígenas de Cotacachi - UNOR(AC). Las muestras se trasladaron inmediatamente a los laboratorios de la Facultad de Ciencias de la Ingeniería de la Universidad Tecnológica Equinoccial (Quito).

\section{Selección de la dosis efectiva}

Para determinar las condiciones experimentales adecuadas, se ensayaron diferentes dosis de luz UV-C. Frutos sin capuchón, seleccionados y lavados, se dividieron en dos grupos: control (no 
irradiados) y tratados (irradiados). Estos últimos se colocaron bajo cuatro lámparas UV-C (TUV G30T8, 30W, Philips) a una distancia de $30 \mathrm{~cm}$ y fueron irradiados con dosis de 8 y $12 \mathrm{~kJ} / \mathrm{m2}$. La intensidad de la radiación fue medida con un radiómetro digital UV (UVX RADIOMETER UVP). Frutos control y tratados se empacaron en bandejas de PVC tipo clamshelf y se almacenaron a $5^{\circ} \mathrm{C}$ durante 28 días. Tres bandejas de frutos control y tratados se retiraron de la cámara de almacenamiento a los 0, 7, 14, 21 y 28 días para evaluar el efecto de cada tratamiento sobre el índice de daño, pérdida de peso y firmeza.

\section{Uso combinado de radiación UV-C y empaque en atmósfera modificada AM}

Una vez elegida la dosis de radiación, frutos no irradiados e irradiados se empacaron en atmósferas modificadas con las siguientes concentraciones de gases: a) 5\% $\mathrm{CO}_{2}$ y $5 \%$ O2; b) 5\% $\mathrm{CO}_{2}$ y $10 \%$ O2. Para el proceso de empacado se utilizó bolsas de polietileno de baja densidad PEBD $(35 \mu \mathrm{m})$ y los gases se dosificaron con una empacadora de alimentos (marca Komet Vacuboy). Finalizado el tratamiento los frutos se almacenaron a $5^{\circ} \mathrm{C}$ durante 28 días. Muestras control (sin ningún tratamiento) se empacaron en bandejas de PVC perforadas tipo clamshelf y se almacenaron directamente a $5^{\circ} \mathrm{C}$. Muestras control y tratadas se retiraron de la cámara de almacenamiento a los 0, 7, 14, 21, 28 y 35 días para evaluar el efecto de cada tratamiento sobre el índice de daño, pérdida de peso y color superficial

Pérdida de peso.- Se pesó cada bandeja al inicio y al término de cada período de almacenamiento. Se determinó la pérdida de peso como porcentaje del peso inicial.

Índice de daño (ID).- Cada día de muestreo los frutos se evaluaron visualmente utilizando una escala subjetiva de 1 a 4 , considerando los siguientes parámetros: decaimiento, picaduras, manchas en la superficie y apariencia, donde: $4=$ daño severo, $3=$ daño moderado, $2=$ daño ligero y $1=\sin$ daño.

Firmeza.- Se midió mediante un penetrómetro de frutas, Tr. Italy, usando una punta de $5 \mathrm{~mm}$ de diámetro.
Color superficial.- El color se midió con un colorímetro de superficie, Konica Minolta, CR410, se utilizó la escala: CIE L*a* b*.

\section{Análisis estadístico}

Se empleó un diseño factorial. Los resultados fueron procesados mediante un ANOVA y las medidas comparadas por la prueba de diferencias mínimas significativas (DMS) con un $\alpha=0,05$.

\section{Resultados y Discusión}

\section{Selección de la dosis efectiva}

Pérdida de peso.- Durante 28 días de almacenamiento en refrigeración a $5^{\circ} \mathrm{C}$, los frutos de uvilla, tanto control como tratados (8 y 12 $\mathrm{kJ} / \mathrm{m} 2$ ) presentaron incremento de la pérdida de peso (Figura 1). Al final del almacenamiento se encontró una pérdida de peso de 21.5, 18.6 y 15.1\% para las muestras control, 8 y $12 \mathrm{~kJ} / \mathrm{m} 2$, respectivamente. La dosis de $12 \mathrm{~kJ} / \mathrm{m} 2$, que fue la que produjo menor pérdida de peso durante el almacenamiento. Es decir que, mientras mayor sea la dosis de radiación UV-C aplicada, se observa menor pérdida de peso, sin embargo la aplicación de altas dosis podría provocar defectos en la apariencia del producto lo que depende de cada sistema biológico (Rivera-Pastrana, Martinez-Tellez, RiveraDominguez, \& González-Aguilar, 2007).

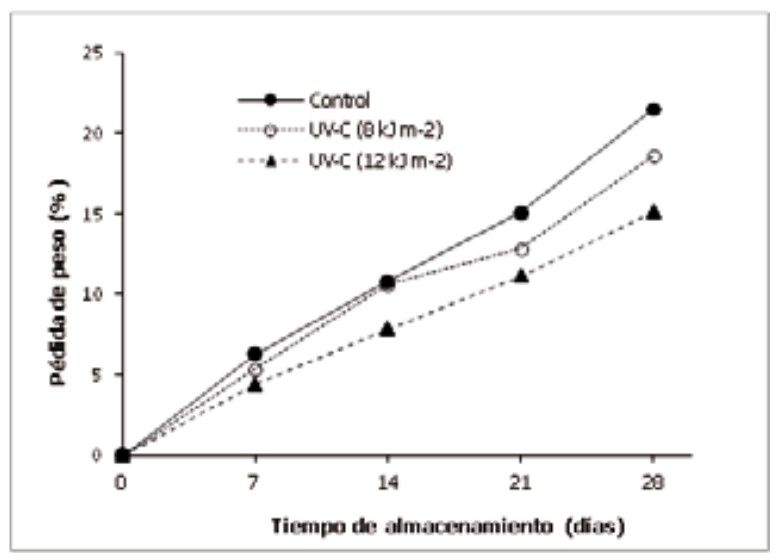

Figura 1. Pérdida de peso de uvilla orgánica en función del tiempo de almacenamiento $\mathrm{a}^{\circ} \mathrm{C}$. DMS $\alpha=0,05=2.89$

Índice de daño (ID).- Durante el almacenamiento los frutos tratados presentaron menor índice de daño que los control. 
Las muestras de uvilla control presentaron síntomas de daño a partir del día 7 con un ID de 1.40, (sin daño - daño ligero), alcanzando en el día 28 un valor de ID=3.52 (daño moderado - daño severo).

Los frutos tratados con la dosis de $12 \mathrm{~kJ} / \mathrm{m} 2$ mostraron síntomas de daño a partir del día 14 con un ID=1.65 que corresponde a sin daño a daño ligero, al final del almacenamiento (día 28) los frutos alcanzaron un valor de ID=2.94 (correspondiente a daño ligero - daño moderado) mientras que los frutos tratados con $8 \mathrm{~kJ} / \mathrm{m} 2$ alcanzaron en el día 14 valores de ID=1.75 (correspondiente a sin daño - daño ligero) y en el día 28 presentaron un ID=3.07, correspondiente a daño moderado (Figura 2).

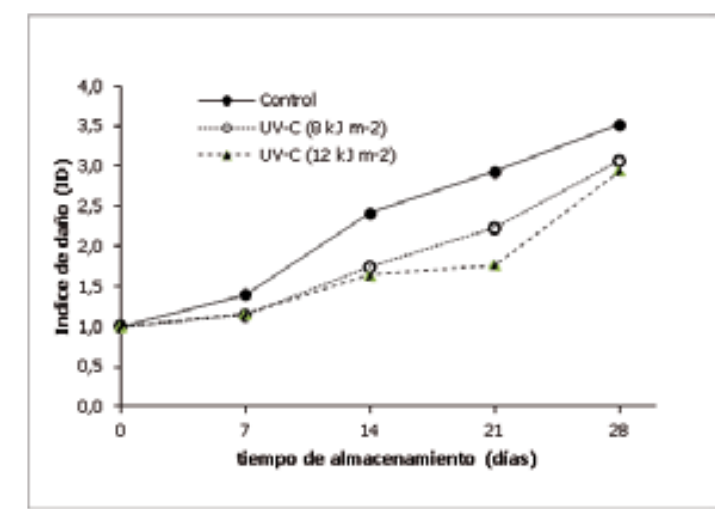

Figura 2. Variación del índice de daño de uvilla orgánica en función del tiempo de almacenamiento $^{\circ} \mathrm{C}$. DMS $\mathrm{\alpha}=0,05=0.29$



Figura 3. Frutos de uvilla, muestras control y tratadas (8 y $12 \mathrm{~kJ} / \mathrm{mz}$ ) durante el tiempo de almacenamiento $a 5^{\circ} \mathrm{C}$.
En la figura 3 se puede observar la evolución del daño, tanto de frutos control como tratados ( 8 y $12 \mathrm{~kJ} / \mathrm{mz}$ ) a lo largo del período de almacenamiento.

Al igual que en el presente estudio, Andrade, Moreno, Henríquez, Gómez, y Concellón (2010) reportaron menor índice de daño para muestras de carambola mínimamente procesada tratada con radiación UV-C.

Firmeza.- Los frutos control presentaron menor firmeza que los frutos tratados a 8 y $12 \mathrm{~kJ} / \mathrm{m} 2$. Para el último día de análisis la muestra tratada con $12 \mathrm{~kJ} / \mathrm{m}^{2}$ tuvo menor firmeza $(5.83 \mathrm{~N})$ en comparación con la muestra control y tratada a $8 \mathrm{~kJ} / \mathrm{m}^{2}$ que presentaron valores de 7.58 y 7.85 $\mathrm{N}$ respectivamente (figura 4). La radiación UV-C influyó de manera positiva en las frutas tratadas en comparación con las frutas no irradiadas que presentaron mayor ablandamiento.

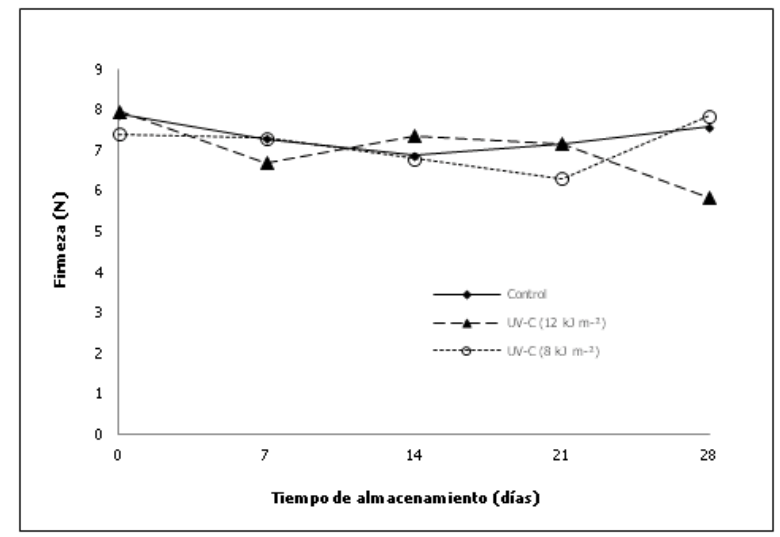

Figura 4. Variación de firmeza en uvilla orgánica en función del tiempo de almacenamiento $5^{\circ} \mathrm{C}$. DMS $\alpha=0,05=0.45$

La firmeza de las frutas estaría relacionada directamente con la integridad de las paredes celulares Baka et al. (1999) reportó que los tratamientos con radiación UV-C podrían reducir la actividad de las enzimas degradantes de la pared celular retrasando el ablandamiento, lo que podría favorecer a mantener la calidad comercial por más días.

Los frutos tratados con $12 \mathrm{~kJ} / \mathrm{m} 2$ presentaron menor pérdida de peso, menor ablandamiento y mejores características visuales durante el periodo de almacenamiento que los frutos control y tratados con $8 \mathrm{~kJ} /$ m2; es decir que la aplicación de $12 \mathrm{~kJ} / \mathrm{m} 2$ permitió mantener la calidad 
comercial de los frutos por más tiempo, por lo que se seleccionó esta dosis para posteriores ensayos.

\section{Uso combinado de radiación UV-C y empaque en atmósfera modificada AM}

Pérdida de peso.- Se produjo un incremento en la pérdida de peso tanto de frutos tratados como controles (Tabla 1). Los frutos control (sin UV-C y sin AM) presentaron la mayor pérdida de peso alcanzando en el día 35 valores de $19.4 \%$ mientras que el empaque en $\mathrm{AM}$ redujo la pérdida de peso a valores entre 2-5\%. Los frutos en los que se aplicó la combinación de la radiación UV-C (12 kJ/m2) y AM presentaron una pérdida de peso de 1.02 y $2.6 \%$ para mezclas de gases de $5 \% \mathrm{CO}_{2}-5 \% \mathrm{O}_{2}$ y $5 \% \mathrm{CO}_{2}-10 \% \mathrm{O} 2$, respectivamente.

\begin{tabular}{|c|c|c|c|c|c|}
\hline \multirow[b]{2}{*}{$\begin{array}{c}\text { Tiempo de } \\
\text { almacenamiento } \\
\text { (días) }\end{array}$} & \multicolumn{5}{|c|}{ Tratamiento } \\
\hline & Control & $\begin{array}{r}5 \% \mathrm{O}_{2^{-}} \\
5 \% \mathrm{CO}_{2} \\
\end{array}$ & $\begin{array}{l}10 \% \mathrm{O}_{2-} \\
5 \% \mathrm{CO}_{2}\end{array}$ & $\begin{array}{c}\text { UV-C + } \\
5 \% \mathrm{O}_{2}-5 \% \mathrm{CO}_{2}\end{array}$ & $\begin{array}{c}\text { UV-C } \\
+10 \% \mathrm{O}_{2-} \\
5 \% \mathrm{CO}_{2}\end{array}$ \\
\hline 7 & 5.42 & 1.11 & 3.24 & 0.59 & 1.66 \\
\hline 14 & 7.59 & 1.53 & 3.82 & 0.76 & 1.96 \\
\hline 21 & 11.57 & 2.26 & 3.92 & 0.92 & 2.24 \\
\hline 28 & 15.07 & 2.74 & 4.69 & 0.93 & 2.45 \\
\hline 35 & 19.37 & 2.94 & 5.87 & 1.02 & 2.57 \\
\hline $\begin{array}{l}\text { DMS }_{a=0,05} \\
\text { Significancia }^{b}\end{array}$ & 1.23 & & & & \\
\hline Tratamiento $(\mathrm{T})$ & sig & & & & \\
\hline Tiempo (t) & sig & & & & \\
\hline Txt & sig & & & & \\
\hline
\end{tabular}

Tabla 1. Pérdida de peso (\%) de uvilla orgánica tratada con radiación UV-C $(12 \mathrm{~kJ} / \mathrm{m} 2)$ y empacada en atmósfera modificada durante el almacenamiento a $5^{\circ} \mathrm{Ca}$

Índice de daño.- Las muestras de uvilla control (sin UV-C y sin AM) presentaron síntomas de daño a partir del día 21 con un ID de 2.20, (daño ligero), alcanzando en el día 35 un valor de ID=3.47 (daño moderado a severo). Los frutos tratados con $\mathrm{AM}$ (5\%CO2-5\% O2 y $5 \% \mathrm{CO}_{2}-10 \% \mathrm{O}_{2}$ ) mostraron síntomas de daño a partir del día 21 y al final del almacenamiento (día 35) alcanzaron un valor de ID=3,00 y 3,27 (correspondiente a daño moderado) para las mezclas de gases de 5\%CO2-5\% O2 y 5\%CO2-10\% O2, respectivamente (figura 5). Mientras que los frutos tratados con $12 \mathrm{~kJ} /$ m2 y empacados en AM alcanzaron en el día 21 valores entre 1,00 y 1,13 (correspondiente a sin daño). En el día 35 presentaron un ID=2,67 y 2,53 (correspondiente a daño ligero - daño moderado) para los tratamientos UV-C+5\%CO2-5\% O2 y UV-C +5\%CO2-10\% O2, respectivamente.

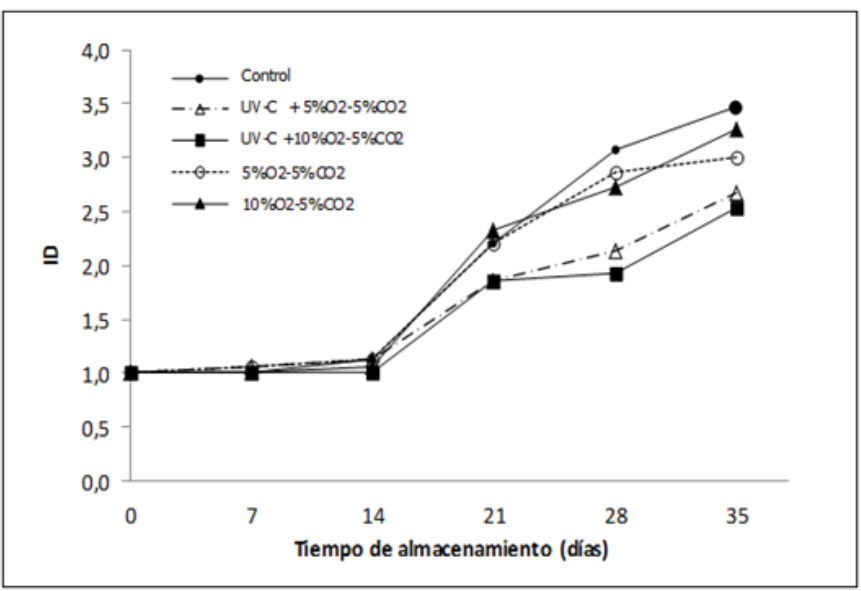

Figura 5. Evolución del ID de uvilla orgánica en función del tiempo de almacenamiento $5^{\circ} \mathrm{C}$. DMSO $0.05=0.33$

La combinación de la radiación UV-C y AM retrasó la aparición de síntomas de daño en uvilla. La principal pérdida poscosecha en este fruto se debealataquefúngico, entonces, la aplicación de untratamiento con efecto fungicida como la radiación UV-C y posteriormente el almacenamiento en AM permitiría controlar el crecimiento de microorganismos de forma que se retrase el deterioro de la fruta y se alargue su vida útil.

Color superficial.- Como se indica en la tabla 2, los valores de L* se incrementaron en todas las muestras hasta el día 14, a partir del cual se mantuvieron constantes para los tratamientos $\left(5 \% \mathrm{O}_{2}-5 \% \mathrm{CO}_{2} ; 10 \% \mathrm{O}_{2}\right.$ 5\%CO2; UV-C+5\%O2-5\%CO2; UV-C+0\%O2-5\%CO2) hasta el día 28, únicamente las muestras control presentaron una disminución del valor de L* a partir del día 21 alcanzando menores valores que las tratadas en el día 35.

Los parámetros Hue, a* y b* de las muestras tratadas $(5 \% \mathrm{O} 2-5 \% \mathrm{CO} 2$; $10 \% \mathrm{O}_{2}-5 \% \mathrm{CO} 2$; UV-C+5\%O2-5\%CO2; UV-C+0\%O2-5\%CO2) se mantuvieron constantes a lo largo del almacenamiento y no presentaron diferencias estadísticamente significativas. Las muestras control presentaron menores valores de Hue y a* que las tratadas durante el almacenamiento, se observó disminución de b* hasta el día 28 a partir del cual se mantuvo constante hasta el final del almacenamiento (día 35). 


\begin{tabular}{|c|c|c|c|c|c|}
\hline $\begin{array}{c}\text { Tiempo } \\
\text { (días) }\end{array}$ & Tratamiento & $\mathbf{L}^{*}$ & a* & b* & Hue \\
\hline \multirow{5}{*}{7} & Control & 63,54 & 16,00 & 53,05 & 73,24 \\
\hline & $5 \% \mathrm{O}_{2}-5 \% \mathrm{CO}_{2}$ & 63,65 & 14,92 & 53,17 & 74,37 \\
\hline & $10 \% \mathrm{O}_{2}-5 \% \mathrm{CO}_{2}$ & 63,05 & 14,55 & 52,51 & 74,51 \\
\hline & UV-C $+5 \% \mathrm{O}_{2}-5 \% \mathrm{CO}_{2}$ & 64,10 & 14,91 & 54,14 & 74,62 \\
\hline & UV-C $+10 \% \mathrm{O}_{2}-5 \% \mathrm{CO}_{2}$ & 62,40 & 14,02 & 51,31 & 74,74 \\
\hline \multirow{5}{*}{14} & Control & 63,95 & 16,66 & 54,02 & 72,88 \\
\hline & $5 \% \mathrm{O}_{2}-5 \% \mathrm{CO}_{2}$ & 66,08 & 15,39 & 54,60 & 74,29 \\
\hline & $10 \% \mathrm{O}_{2}-5 \% \mathrm{CO}_{2}$ & 66,37 & 15,22 & 54,20 & 74,38 \\
\hline & UV-C $+5 \% \mathrm{O}_{2}-5 \% \mathrm{CO}_{2}$ & 65,58 & 14,98 & 53,94 & 74,53 \\
\hline & UV-C $+10 \% \mathrm{O}_{2}-5 \% \mathrm{CO}_{2}$ & 64,01 & 13,96 & 52,24 & 75,06 \\
\hline \multirow{5}{*}{21} & Control & 61,70 & 17,38 & 51,65 & 71,38 \\
\hline & $5 \% \mathrm{O}_{2}-5 \% \mathrm{CO}_{2}$ & 66,61 & 16,70 & 55,25 & 73,21 \\
\hline & $10 \% \mathrm{O}_{2}-5 \% \mathrm{CO}_{2}$ & 66,45 & 16,30 & 54,46 & 73,36 \\
\hline & UV-C $+5 \% \mathrm{O}_{2}-5 \% \mathrm{CO}_{2}$ & 63,87 & 14,66 & 52,08 & 74,36 \\
\hline & UV-C $+10 \% \mathrm{O}_{2}-5 \% \mathrm{CO}_{2}$ & 65,04 & 15,00 & 53,20 & 74,27 \\
\hline \multirow{5}{*}{28} & Control & 58,22 & 17,20 & 49,38 & 70,84 \\
\hline & $5 \% \mathrm{O}_{2}-5 \% \mathrm{CO}_{2}$ & 63,27 & 14,24 & 52,05 & 74,76 \\
\hline & $10 \% \mathrm{O}_{2}-5 \% \mathrm{CO}_{2}$ & 63,50 & 14,40 & 52,12 & 74,59 \\
\hline & UV-C $+5 \% \mathrm{O}_{2}-5 \% \mathrm{CO}_{2}$ & 62,38 & 15,31 & 51,96 & 73,61 \\
\hline & UV-C $+10 \% \mathrm{O}_{2}-5 \% \mathrm{CO}_{2}$ & 63,09 & 15,07 & 52,28 & 73,94 \\
\hline \multirow{9}{*}{35} & Control & 60,62 & 17,24 & 49,91 & 70,94 \\
\hline & $5 \% \mathrm{O}_{2}-5 \% \mathrm{CO}_{2}$ & 66,95 & 16,55 & 55,05 & 73,38 \\
\hline & $10 \% \mathrm{O}_{2}-5 \% \mathrm{CO}_{2}$ & 66,76 & 15,92 & 53,88 & 73,54 \\
\hline & UV-C $+5 \% \mathrm{O}_{2}-5 \% \mathrm{CO}_{2}$ & 64,81 & 15,40 & 52,30 & 73,63 \\
\hline & UV-C $+10 \% \mathrm{O}_{2}-5 \% \mathrm{CO}_{2}$ & 64,50 & 15,61 & 52,75 & 73,54 \\
\hline & $\begin{array}{l}\mathrm{DMS}_{0.05} \\
\text { Significancia }\end{array}$ & 1,55 & 1,09 & 1,70 & 1,83 \\
\hline & Tratamiento $(\mathrm{T})$ & sig & sig & sig & sig \\
\hline & Tiempo $(t)$ & sig & sig & sig & sig \\
\hline & Txt & sig & sig & sig & sig \\
\hline
\end{tabular}

a Media ( $n=30)$

$\mathrm{b}$ sig = diferencia significativa a $\mathrm{p}<0.05$

Tabla 2. Luminosidad ( $\left.L^{*}\right), a^{*}, b^{*}$ y ángulo de tono (Hue) en frutos control y tratados durante el almacenamiento a $5^{\circ} \mathrm{Ca}$

Normalmente en las frutas, los cambios en color son controlados por enzimas que dependen del oxígeno y que regulan la síntesis de $\beta-$ caroteno (López Valenzuela, Valverde Juárez, Mejía Torres, López Angulo, \& Vega García, 2011). En el presente estudio, probablemente el uso de AM influyó sobre la actividad de estas enzimas y se produjo ligeros cambios en el color debido al bajo oxígeno. Sin embargo hacen falta estudios para dilucidar el mecanismo de acción de la radiación UV-C y AM sobre el desarrollo del color en la uvilla.

Los ligeros cambios observados podrían deberse a que, según explican Martín-Belloso y Oms-Oliu (2005), las atmósferas bajas en $\mathrm{O} 2$ y altas en $\mathrm{CO}_{2}$ pueden disminuir la velocidad de pardeamiento de los tejidos vegetales. Esta mejora en el color va acompañada por diversos procesos fisiológicos como la disminución de la tasa de respiración, un retraso del pico climatérico con la consiguiente producción de etileno, y una maduración más lenta. Estos procesos, a su vez, se relacionan con la senescencia de los tejidos y, consecuentemente, con la liberación de enzimas y sustratos que participan en las reacciones de pardeamiento.

\section{Conclusiones}

Se seleccionó la dosis de $12 \mathrm{~kJ} / \mathrm{m} 2$ porque produjo menor pérdida de peso, índice de daño y ablandamiento, permitiendo mantener la calidad comercial de los frutos por más tiempo (durante 28 días) que los frutos control y tratados con $8 \mathrm{~kJ} / \mathrm{m2}$.

El uso combinado de la radiación UV-C (12 kJ/m2) y el empaque en $\mathrm{AM}\left(5 \% \mathrm{CO}_{2}-5 \% \mathrm{O} 2\right.$ y 5\%CO2-10\% O2) redujo la pérdida de peso y produjo cambios en los parámetros L* y Hue de la uvilla. Entre las mezclas de gases ensayadas, fue la concentración 5\%CO2-5\%O2 en combinación con luz UV-C la que retrasó la aparición de síntomas de daño permitiendo incrementar la vida útil de la uvilla hasta 35 días. Los frutos tratados con radiación UV-C y almacenados en AM conservaron mejores características que los frutos control y los almacenados directamente en AM.

Se requiere generar conocimiento más profundo de los procesos fisiológicos y bioquímicos que se producen en el interior del envase tras el tratamiento UV-C y de los factores que intervienen en el control de la atmósfera como estado de madurez, peso del producto, temperatura, entre otros. La modelización de estos procesos podría permitir establecer condiciones óptimas de conservación en AM y la selección o diseño de envases adecuados. 


\section{Agradecimientos}

- Dirección de Investigación y Transferencia Tecnológica ITT. Proyecto de investigación: "Uso combinado de la radiación UV-C y atmósfera modificada para disminuir el deterioro de uvilla (Physalis peruviana L.) de exportación”. Facultad de Ciencias de la Ingeniería. Universidad Tecnológica Equinoccial. Quito-Ecuador.

- Empresa Sumak Mikuy. Unión de Organizaciones Campesinas e Indígenas de Cotacachi, provincia de Imbabura UNORCAC. Cotacachi-Ecuador.

\section{Bibliografía}

Andrade, M. J, Moreno, C, Henríquez, A, Gómez, A, \& Concellón, A. (2010). Influencia de la Radiación UV-C como Tratamiento Postcosecha sobre Carambola (Averroha carambola L.) Mínimamente Procesada Almacenada en Refrigeración. Revista Iberoamericana de Tecnología Postcosecha, 11.

Baka, M, Mecier, J, Corcuff, F, Castaigne, F, \& Arual, J. (1999). Photochemical treatment to improve storability of fresh strawberries. J. Food Sci, 64, 1068-1072.

Brito, D. (2002). Agroexportación de productos no tradicionales. Producción de uvilla para exportación: Fundación Aliñambi.

CORPEI. (2009). Centro de información e Inteligencia comercial. Perfil de Uvilla. Retrieved from www.pucesi.edu.ec/pdf/ uvilla.pdf.

García, H. R, Peña, A. C, \& García, C. (2008). Manual de prácticas de cosecha y acondicionamiento de la uchuva con fines de exportación Corpoica. Bogotá, Colombia.

Herrera, A. (2003). Manejo Postcosecha. In: . In V. Flores, G. Fisher \& A. Sora (Eds.), Producción, Postcosecha y Exportación de Uchuva (Physalis peruviana L.). Colombia: Universidad Nacional de Colombia. Facultad de Agronomía.

López, E, \& Páez, G. (2002). Comportamiento fisiológico de la uchuva (Physalis peruviana L.) bajo condiciones de refrigeración y películas plásticas para su conservación y poscosecha. (Trabajo de grado), Universidad Nacional de Colombia, Bogotá.

López Valenzuela, José Ángel, Valverde Juárez, Francisco Javier, Mejía Torres, Silvia Lizzeth, López Angulo, Gabriela, \& Vega García, Misael Odín. (2011). Efecto del almacenamiento en atmósfera controlada sobre la calidad poscosecha y nutricional del tomate. Revista Chapingo. Serie horticultura, 17(2), 115-128.

Martín-Belloso, O, \& Oms-Oliu, G. (2005). Efecto de la atmósfera modificada e las características físico químicas y nutricionales de la fruta fresca cortada Proyecto X1.22 Desarrollo de tecnologías para la conservación de vegetales cortados. La Habana-Cuba.

PROFIAGRO. (2007). Estudio de Factibilidad uvilla. Retrieved 15 de Agosto de 2009, from http://www.corpei.org/archivos/file/ profiagro/descargas/uvilla-estudio-prefactibilidad.pdf

Rivera-Pastrana, D, Martinez-Tellez, M, Rivera-Dominguez, M, \& González-Aguilar, G. (2007). Efectos bioquímicos poscosecha de la irradiacion UV-C en frutas y hortalizas. Fiotecnia Mexicana, 30(004), 361-372.

Sánchez, J. (2002). Esc. Agríc. Panamericana. El Zamorano.

Stevens, C, Khan, V. A, Lu, J. Y, Wilson, C. L, Pusey, P. L, \& Igwegbe, E. C. K. (1997). Integration of ultraviolet (UV-C) light with yeast treatment for control of postharvest storage rots of fruits an vegetables. Biol. Control, 10, 98-103. 
